\title{
Optimal Trajectory Design of Formation Flying based on Attractive Sets
}

\author{
By Motoki Yamane, ${ }^{1)}$ Mai BAndo, ${ }^{1)}$ and Shinji HoKAmoto ${ }^{1)}$ \\ ${ }^{1)}$ Department of Aeronautics and Astronautics, Kyushu University, Fukuoka, Japan
}

(Received June 23rd, 2017)

\begin{abstract}
This paper presents a new method of optimal trajectory design for formation flying. Under linearized assumptions and a quadratic performance index, we introduce an attractive set of optimal control based on the linear quadratic regulator theory. The attractive set is defined as a set of all initial states to reach a desired state for a given cost. In particular, we consider attractive sets for two problems: a fixed final-state, fixed final-time problem and an infinite-time problem, and the optimal initial state is found based on the geometry of the attractive set. The optimal trajectories for two problems are evaluated in terms of $L_{1}$-norm of control input and termination time.
\end{abstract}

Key Words: Spacecraft, Formation Flying, Optimal Control

$\begin{array}{cll}\text { Nomenclature } & & \\ A & : & \text { state matrix } \\ B & : & \text { input matrix } \\ C & : & \text { a constant } \\ J & : & \text { performance index } \\ J^{*} & : & \text { minimum value of performance index } \\ n & : & \text { mean motion } \\ Q, R & : & \text { weight matrices } \\ \boldsymbol{R}_{c} & : & \text { radius vector of chaser orbit } \\ \boldsymbol{R}_{0} & : & \text { radius vector of target orbit } \\ T & : & \text { orbital period } \\ \boldsymbol{u} & : & \text { control input vector } \\ \boldsymbol{u}^{*} & : & \text { optimal control input vector } \\ \boldsymbol{x} & : & \text { state vector } \\ \boldsymbol{X} & : & \text { coefficient matrix of attractive set } \\ x, y, z & : & \text { position } \\ \boldsymbol{\varepsilon} & : & \text { acceptable error range } \\ \mu & : & \text { geocentric gravity constant } \\ \text { Subscripts } & : & \text { initial } \\ 0 & : & \text { final } \\ \mathrm{f} & : & \text { optimal } \\ \text { opt } & & \end{array}$

\section{Introduction}

The Hill's equations (also known as the Hill-ClohessyWiltshire (HCW) equations) are equations of relative motion of a chaser linearized around a target. ${ }^{1}$ The Hill's equations have periodic solutions that are convenient for formation flying. The rendezvous problem for a target on a circular orbit has been studied extensively in the literature. ${ }^{2)}$ The optimal rendezvous problem using continuous thrust is often treated as the optimal control problem. In designing a feedback control for a rendezvous problem, a linear quadratic regulator is often used., ${ }^{3,4)}$ A linear quadratic regulator is a control law that minimizes a quadratic performance index for a linear system. Once the initial and final states are specified, the optimal trajectory can be obtained based on linear quadratic regulator theory. However, when using this method, it is necessary to solve the two-point boundary-value problem (TPBVP) repeatedly to determine the optimal initial state. To solve the TPBVP for rendezvous prob- lem, the generating functions which give the optimal control as a function of initial and final state are used to find the optimal initial state in Refs. 5) and 6).

We present a new method of optimal trajectory design for formation flying. Under linearized assumptions and a quadratic performance index, we introduce an attractive set of optimal control based on linear quadratic regulator theory. The attractive set is defined as a set of all initial states to reach a desired state for a given cost. Using the attractive set for optimal trajectory design, we can determine the optimal initial states among possible solutions.

In a real mission, it is desirable to complete an orbit transfer in finite time. Therefore, this paper reveals the relation between the shape of an attractive set and an optimal trajectory for different boundary conditions. First, we define attractive sets for two optimal control problems: a fixed final-state, fixed finaltime problem and an infinite-time problem, then we propose a novel method for finding the optimal initial state based on the attractive set. Next, by computing the contour of the an attractive set on a chaser's initial orbit, we discuss the shape of the attractive set with the trajectory when termination time is increased. Moreover, we evaluate the obtained trajectory using the $L_{1}$-norm of the control input and compare the $L_{1}$-norm with the termination time. For the infinite-time problem, an interesting property of the attractive set is also shown.

\section{Equations of Motion}

We consider relative motion between a target and a chaser on a circular orbit. Figure 1 shows the chaser's equations of motion relative to a target assuming the coordinate system $(x, y, z)$ as follows:

$$
\begin{aligned}
& \ddot{x}=2 n \dot{y}+n^{2}\left(R_{0}+x\right)-\frac{\mu}{R^{3}}\left(R_{0}+x\right)+u_{x} \\
& \ddot{y}=-2 n \dot{x}+n^{2} y-\frac{\mu}{R^{3}} y+u_{y} \\
& \ddot{z}=-\frac{\mu}{R^{3}} z+u_{z}
\end{aligned}
$$

When these equations are linearized at the origin, Eqs. (1) to (3) become 


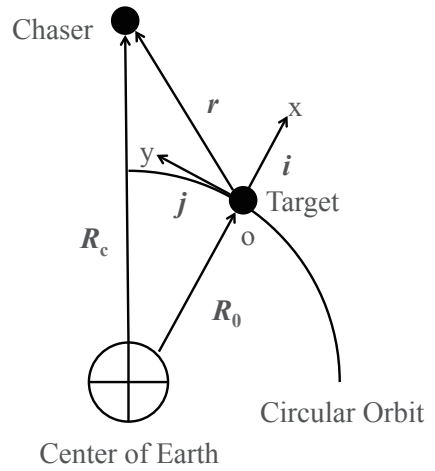

Fig. 1. Definition of coordinate system.

$$
\begin{aligned}
& \ddot{x}=3 n^{2} x+2 n \dot{y}+u_{x} \\
& \ddot{y}=-2 n \dot{x}+u_{y} \\
& \ddot{z}=-n^{2} z+u_{z}
\end{aligned}
$$

These equations are called Hill's equations. ${ }^{1)}$

Let $\boldsymbol{x}=[x, y, z, \dot{x}, \dot{y}, \dot{z}]^{\mathrm{T}}$ and $\boldsymbol{u}=\left[u_{x}, u_{y}, u_{z}\right]^{\mathrm{T}}$ be a state vector and an input vector, respectively. The Hill's equations can then be represented by

$$
\dot{x}=A x+B u, \quad x(0)=x_{0}
$$

where

$$
\begin{aligned}
A & =\left(\begin{array}{rrrrrr}
0 & 0 & 0 & 1 & 0 & 0 \\
0 & 0 & 0 & 0 & 1 & 0 \\
0 & 0 & 0 & 0 & 0 & 1 \\
3 n^{2} & 0 & 0 & 0 & 2 n & 0 \\
0 & 0 & 0 & -2 n & 0 & 0 \\
0 & 0 & -n^{2} & 0 & 0 & 0
\end{array}\right) \\
B & =\left(\begin{array}{lll}
0 & 0 & 0 \\
0 & 0 & 0 \\
0 & 0 & 0 \\
1 & 0 & 0 \\
0 & 1 & 0 \\
0 & 0 & 1
\end{array}\right)
\end{aligned}
$$

For simplicity, we only consider in-plane motion. Solving Eqs. (4) and (5) under $\boldsymbol{x}_{0}=\left[x_{0}, y_{0}, \dot{x}_{0}, \dot{y}_{0}\right]^{\mathrm{T}}, \boldsymbol{u}=0$ yields

$$
\begin{aligned}
x= & \left(-3 x_{0}-\frac{2}{n} \dot{y}_{0}\right) c+\frac{\dot{x}_{0}}{n} s+4 x_{0}+\frac{2}{n} \dot{y}_{0} \\
y= & \frac{2}{n} \dot{x}_{0} c+\left(6 x_{0}+\frac{4}{n} \dot{y}_{0}\right) s \\
& +\left(-6 n x_{0}-3 \dot{y}_{0}\right) t+y_{0}-\frac{2}{n} \dot{x}_{0} \\
\dot{x}= & \dot{x}_{0} c+\left(3 n x_{0}+2 \dot{y}_{0}\right) s \\
\dot{y}= & 2\left(3 n x_{0}+2 \dot{y}_{0}\right) c-2 \dot{x}_{0} s \\
& -3\left(2 n x_{0}+\dot{y}_{0}\right)
\end{aligned}
$$

where $c \equiv \cos n t, \quad s \equiv \sin n t$. From Eqs. (10) to (13), in the case where the secular term is equal to zero, a periodic solution is obtained:

$$
\dot{y}_{0}=-2 n x_{0}
$$

Under this condition, Eqs. (10) to (13) express an elliptic orbit. On this elliptic orbit, the velocity $(\dot{x}, \dot{y})$ is expressed as a function of the position $(x, y)$ :

$$
\begin{aligned}
& \dot{x}=\frac{n}{2} y \\
& \dot{y}=-2 n x
\end{aligned}
$$

\section{Problem Statement and Definition of an Attractive Set}

In this section, the optimal control problem under two types of boundary conditions are reviewed ${ }^{7 / 8) 9)}$ and the attractive set of optimal control is introduced.

\subsection{Fixed final-state, fixed final-time problem}

Consider an optimal control problem for Eq. (7) that minimizes the performance index $J$ given by

$$
J=\int_{t_{0}}^{t_{f}}\left(\boldsymbol{x}^{\mathrm{T}} Q \boldsymbol{x}+\boldsymbol{u}^{\mathrm{T}} R \boldsymbol{u}\right) d t
$$

where $Q \geq 0, R>0$ and $\boldsymbol{x}\left(t_{f}\right)=\boldsymbol{x}_{f}$. The optimal control input that minimizes the performance index is given by

$$
\boldsymbol{u}^{*}=-R^{-1} B^{\mathrm{T}}\left\{S(t) \boldsymbol{x}-U(t) W^{-1}\left(t_{0}\right)\left(U^{\mathrm{T}}\left(t_{0}\right) \boldsymbol{x}_{0}-\boldsymbol{x}_{f}\right)\right\}
$$

where $S, U$, and $W$ are positive-definite solutions that satisfy the following differential equations:

$$
\begin{aligned}
& \dot{S}+A^{\mathrm{T}} S+S A-S B R^{-1} B^{\mathrm{T}} S+Q=0 \\
& \dot{U}=-\left(A^{\mathrm{T}}-S B R^{-1} B^{\mathrm{T}}\right) U \\
& \dot{W}=U^{\mathrm{T}} B R^{-1} B^{\mathrm{T}} U \\
& S\left(t_{f}\right)=\boldsymbol{O}, \quad U\left(t_{f}\right)=\boldsymbol{I}, \quad W\left(t_{f}\right)=\boldsymbol{O}
\end{aligned}
$$

Then the minimum value of the performance index $J^{*}$ is given by

$$
\begin{aligned}
J^{*}= & x_{0}^{\mathrm{T}} S\left(t_{0}\right) x_{0} \\
& -\left(\boldsymbol{x}_{0}^{\mathrm{T}} U_{0}-\boldsymbol{x}_{f}^{\mathrm{T}}\right) W^{-1}\left(t_{0}\right)\left(U_{0}^{\mathrm{T}} \boldsymbol{x}_{0}-\boldsymbol{x}_{f}\right)
\end{aligned}
$$

Equation (23) forms an $n$-dimensional ellipsoid. Consider the set of all initial state inside the $n$-dimensional ellipsoid $J^{*}=C$

$$
\begin{aligned}
\mathscr{A}(C) & =\left\{\boldsymbol{x}_{0} \in \boldsymbol{R}^{n} \mid \boldsymbol{x}_{0}^{\mathrm{T}} S\left(t_{0}\right) \boldsymbol{x}_{0}\right. \\
& \left.-\left(\boldsymbol{x}_{0}^{\mathrm{T}} U_{0}-\boldsymbol{x}_{f}^{\mathrm{T}}\right) W^{-1}\left(t_{0}\right)\left(U_{0}^{\mathrm{T}} \boldsymbol{x}_{0}-\boldsymbol{x}_{f}\right) \leq C\right\}
\end{aligned}
$$

We define $\mathscr{A}(C)$ as the attractive set of optimal control. The optimal trajectory departing from the inside of this ellipsoid is guaranteed that the value of the performance index is less than C.

\subsection{Infinite-time problem}

Consider an optimal control problem for Eq. (7) that minimizes the performance index $J$ given by

$$
J=\int_{t_{0}}^{\infty}\left(\boldsymbol{x}^{\mathrm{T}} Q \boldsymbol{x}+\boldsymbol{u}^{\mathrm{T}} R \boldsymbol{u}\right) d t
$$

where $Q \geq 0, R>0$. The optimal control input that minimizes the performance index is as follows:

$$
\boldsymbol{u}^{*}=-R^{-1} B^{\mathrm{T}} S \boldsymbol{x}
$$

where $S$ is a positive definite solution satisfying the algebraic Riccati equation:

$$
A^{\mathrm{T}} S+S A-S B R^{-1} B^{\mathrm{T}} S+Q=0
$$




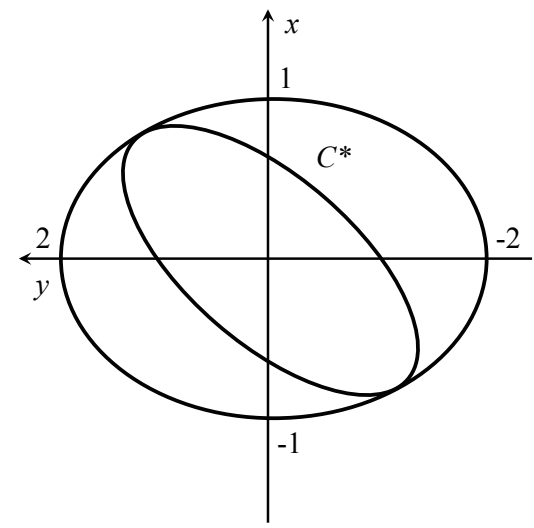

(a) Hill ellipse $\left(x^{2}+\frac{y^{2}}{4}=1\right)$ and attractive set $\boldsymbol{x}_{0}^{\mathrm{T}} S\left(t_{0}\right) \boldsymbol{x}_{0}=C^{*}$.

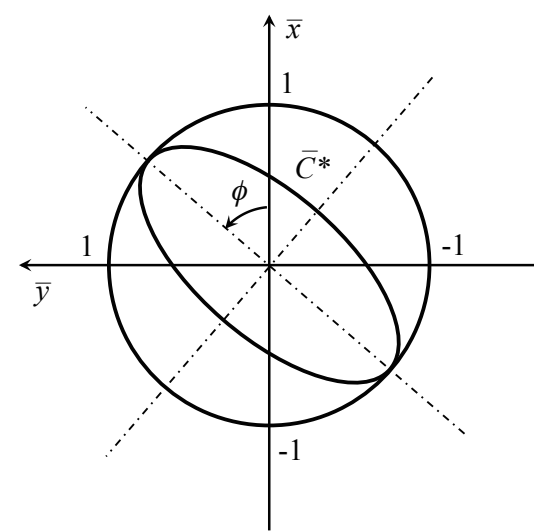

(b) Converted Hill ellipse $\left(\bar{x}^{2}+\bar{y}^{2}=1\right)$ and $\overline{C^{*}}$.

Fig. 2. Conversion of Hill ellipse to a true circle through the coordinate transformation from $(x, y)$ to $(\bar{x}, \bar{y})$.

Then the minimum value of the performance index $J^{*}$ is as follows:

$$
J^{*}=\boldsymbol{x}_{0}^{\mathrm{T}} S\left(t_{0}\right) \boldsymbol{x}_{0}
$$

The attractive set for optimal control is defined as

$$
\mathscr{A}(C)=\left\{x_{0} \in \boldsymbol{R}^{n} \mid \boldsymbol{x}_{0} S\left(t_{0}\right) \boldsymbol{x}_{0} \leq C\right\}
$$

In this case, the coefficient matrix $X$ is simply given by the solution of the Riccati equation $S\left(t_{0}\right)$.

\subsection{Optimal trajectory design by using attractive set}

Let $\boldsymbol{x}_{f}=\mathbf{0}$ to express the simple form of the attractive set in the following discussion. In this case, the attractive set (24) becomes

$$
\mathscr{A}(C)=\left\{\boldsymbol{x}_{0} \in \boldsymbol{R}^{n} \mid \boldsymbol{x}_{0}^{\mathrm{T}} X \boldsymbol{x}_{0} \leq C\right\}
$$

where $X \triangleq S\left(t_{0}\right)-U_{0} W^{-1}\left(t_{0}\right) U_{0}^{\mathrm{T}} . X$ is called the coefficient matrix of the attractive set in this paper. ${ }^{10)}$ By using the attractive set, it is possible to derive the optimal initial state. It is very effective to represent the attractive set geometrically to obtain the optimal initial position. The distribution of the value of the performance index increases from the inside to the outside. Hence, the optimal initial state is the tangent point of the largest attractive set inscribed in the initial periodic orbit (Hill ellipse). The analytical derivation of the tangent point between attractive set and Hill ellipse is shown below.
The difficulty of this problem is that the size of the attractive set, which is tangent to the Hill ellipse, is unknown. Therefore, it is necessary to determine the size of the attractive set which is tangent to the Hill ellipse. To overcome this difficulty, a coordinate transformation is introduced so that Hill ellipse becomes a true circle. Observe that an ellipse is inscribed in a true circle at vertices, then the optimal initial state can be determined by the following calculations.

Consider the coordinate transformation $(x, y)=(\bar{x}, 2 \bar{y})$ in order to convert Hill ellipse into a true circle shown in Fig. 2. Let the size of the attractive set which is tangent to the periodic orbit be $C^{*}$,

$$
X_{11}^{\prime} x^{2}+2 X_{12}^{\prime} x y+X_{22}^{\prime} y^{2}=C^{*}
$$

where $X^{\prime}=\left[\begin{array}{ll}X_{11}^{\prime} & X_{12}^{\prime} \\ X_{21}^{\prime} & X_{22}^{\prime}\end{array}\right]$ is the coefficient matrix of the attractive set under the periodic condition (see Appendix B). Then, $C^{*}$ implies the minimum or maximum values of the performance index on the periodic orbit. Substituting $(x, y)$ in Eq. (31) by $(\bar{x}, 2 \bar{y})$ yields

$$
\left(\frac{X_{11}^{\prime}}{C^{*}}\right) \bar{x}+2\left(\frac{2 X_{12}^{\prime}}{C^{*}}\right) \overline{x y}+\left(\frac{4 X_{22}^{\prime}}{C^{*}}\right) \bar{y}^{2}=1
$$

This is the equation of $\overline{C^{*}}$ in Fig. 2(b). From Fig. 2(b), the vertices of the attractive set become a tangent point with the circle. Therefore, the angle of the major axis of the attractive set in the new coordinate is

$$
\phi=\frac{1}{2} \arctan \frac{2\left(\frac{2 X_{12}^{\prime}}{C^{*}}\right)}{\left(\frac{4 X_{22}^{\prime}}{C^{*}}\right)-\left(\frac{X_{11}^{\prime}}{C^{*}}\right)}=\frac{1}{2} \arctan \frac{4 X_{12}^{\prime}}{4 X_{22}^{\prime}-X_{11}^{\prime}}
$$

where $\phi \in\left[-\frac{\pi}{4}, \frac{\pi}{4}\right]$ and the optimal initial positions on the circle are given by

$$
\left(\overline{x_{\mathrm{opt}}}, \overline{y_{\mathrm{opt}}}\right)=( \pm \cos \phi, \pm \sin \phi)
$$

Finally, the optimal initial positions described in the original coordinate $\left(\overline{x_{\mathrm{opt}}}=x_{\mathrm{opt}}, \overline{y_{\mathrm{opt}}}=y_{\mathrm{opt}} / 2\right)$ are given by

$$
\left(x_{\mathrm{opt}}, y_{\mathrm{opt}}\right)=( \pm \cos \phi, \pm 2 \sin \phi)
$$

Note that the solution is double-sign corresponds.

Moreover, the length of the major axis of the attractive set is equal to the radius of the circle in the new coordinate. Therefore, the following equation is derived by setting $a=X_{11}^{\prime} / C^{*}, b=2 X_{12}^{\prime} / C^{*}, c=4 X_{22}^{\prime} / C^{*}$ and $A=1$ in Eq. (A5) (see Appendix A):

$$
\sqrt{\frac{2}{\left(\frac{X_{11}^{\prime}}{C^{*}}\right)+\left(\frac{4 X_{22}^{\prime}}{C^{*}}\right)-\sqrt{\left[\left(\frac{X_{11}^{\prime}}{C^{*}}\right)-\left(\frac{4 X_{22}^{\prime}}{C^{*}}\right)\right]^{2}+4\left(\frac{2 X_{12}^{\prime}}{C^{*}}\right)^{2}}}}=1
$$

Then, Eq. (36) is rearranged to a quadratic equation of $C^{*}$ as

$$
C^{* 2}-\left(X_{11}^{\prime}+4 X_{22}^{\prime}\right) C^{*}+4 X_{11} X_{22}-4 X_{12}^{2}=0
$$

It leads to

$$
\begin{aligned}
C^{*}= & \frac{1}{2} X_{11}^{\prime}+2 X_{22}^{\prime} \\
& \pm \frac{1}{2} \sqrt{X_{11}^{\prime 2}-8 X_{11}^{\prime} X_{22}^{\prime}+16 X_{12}^{\prime 2}+16 X_{22}^{\prime 2}}
\end{aligned}
$$


The positive and negative sign correspond to the minimum value and the maximum value of $C$ along the periodic orbit, respectively.

For the general problem with arbitrary terminal state $\left(\boldsymbol{x}\left(t_{f}\right) \neq\right.$ 0), the solution procedure presented in this subsection cannot be used. However, the optimal initial state is still the contact point between two ellipses so that we can obtain the optimal initial state by solving fourth order algebraic equation.

\section{Simulation Results}

We use the non-dimensional form of equations of in-plane motion where Eq. (7) is normalized with $\boldsymbol{R}_{0}$ and $T\left(=\sqrt{\frac{\mu}{\boldsymbol{R}_{0}^{3}}}\right)$. Namely,

$$
\begin{aligned}
A & =\left(\begin{array}{rrrr}
0 & 0 & 1 & 0 \\
0 & 0 & 0 & 1 \\
3 & 0 & 0 & 2 \\
0 & 0 & -2 & 0
\end{array}\right) \\
B & =\left(\begin{array}{ll}
0 & 0 \\
0 & 0 \\
1 & 0 \\
0 & 1
\end{array}\right)
\end{aligned}
$$

We design a feedback control to achieve the rendezvous with a target at the origin by the optimal control described in Sec. 3: a fixed final-state, fixed final-time problem and infinite-time problem. For these two optimal control problems, we show the contour of attractive sets where the initial state of the chaser is given by a periodic orbit. In simulations, a specific trajectory starting from $\boldsymbol{x}_{0}=[1.0,0,0,-2.0]^{\mathrm{T}}$ is shown. Let the initial maneuver time be $t_{0}$ and the termination time be $t_{f}$.

\subsection{Basic properties of attractive sets for finite-time prob- lem}

For the finite-time problem, we set $\boldsymbol{x}_{f}=[0,0,0,0]^{\mathrm{T}}, t_{f}=3,6$ and 9. Let $Q=10^{q} \boldsymbol{I}_{4 \times 4}, R=\boldsymbol{I}_{2 \times 2}$, and change the weight of the state by decreasing the value of $q$. Figures 3 and 4 show the contour of the attractive set (24) (multicolored ellipses) and the optimal trajectory (red line).

First, the general properties of the optimal trajectory obtained by a linear quadratic regulator is investigated. Paying attention to the red line in Figs. 3 and 4, it is found that changing the weight parameter of the state changes the trajectory from straight to spiral.

For the in-plane motion, the attractive set is a fourdimensional ellipsoid of position and velocity. However, if the chaser's initial state is restricted on periodic orbits, the velocity of the chaser is a function of position through Eqs. (15) and (16). Hence, the intersection of the ellipsoid and subspace spanned by periodic orbits is a two-dimensional ellipsoid, and can be drawn as an ellipse on the $x-y$ plane. By plotting this ellipsoid on $x-y$ plane, the value of the performance index along a periodic orbit can be determined uniquely. Figures 3 and 4 show that an attractive set has a distribution such that the value of the performance index increases from the inside to the outside of the ellipse. Moreover, Fig. 6(a) shows the values of $J^{*}$ as a function of initial position $y$ correspoding to Fig. 3(c). In Fig. 6(a), $J^{*}$ takes its minimum values at $y= \pm 2$. From Figs. 3(c) and 6(a), it is also found that the minimum values of the performance index occur at tangent points between the periodic orbit and the ellipse corresponding to the contour of $J^{*}$.

The advantage of using the attractive set is that the optimal initial state among the possible set of initial state are immediately determined. Once we obtain the solution of the Riccati equation by solving Eqs. (19) to (21) (Eq. (27) in the case of the infinite-time problem) we can obtain the optimal initial state immediately. The optimal initial position analytically derived by Eq. (34) is shown in Sec 4.4.

\subsection{Basic properties of attractive sets for infinite-time problem}

Figure 5 shows the contour of the attractive set (29) of the infinite-time problem for the different weight parameters. The properties of the attractive set described in 4.1 hold for the infinite-time problem. Moreover, the attractive set for the infinite-time problem for small $q$ has an interesting feature. In Fig. 5, it is observed that the contour of the attractive set converges to 2:1 ellipse (Hill ellipse) as $Q \rightarrow 0$ and the value of $J^{*}$ is almost constant. This means that the $J^{*}$ is constant wherever the chaser departs from the initial periodic orbit. Moreover, Fig. 6(c) shows the values of $J^{*}$ by changing the initial position $y$ on the periodic orbit corresponding to Fig. 5(c). It is confirmed that the value of $J^{*}$ is almost constant on the periodic orbit from Fig. 6(c). The smaller the weight of the state, the closer the trajectory is to the solution of the Hill's equations, which do not use control input.

This interesting property of attractive set can also be observed for different situation. Consider the attractive set for infinite-time problem when the initial velocity of the chaser is zero, i.e. $x_{0}=[x, y, 0,0]$. Note that the solutions starting from $x_{0}=[x, y, 0,0]$ become drift motion along $y$-axis from Eq. (11). Figure 7 shows the contour of the attractive set for different weight parameters. The attractive set becomes unbounded in $y$-direction as $Q \rightarrow 0$. This is because the chaser can approach the origin by using the drift motion along $y$-axis. In Fig. 7, the optimal trajectory seems to exploit the drift motion along $y$-axis for small $q$.

From the asymptotic behavior of the attractive set for the two situations, it can be said that the attractive set for small $q$ reflects the dynamical structure of the motion since the optimal control exploits the natural motion of the system to reduce the control effort.

\subsection{Discussion of a shape of an attractive set}

For the LQR problem, the weight matrices of the performance index determine the relative importance between state and control input. From Figs. 3, 4 and 5, it can be seen that the optimal trajectory and the shapes of the attractive sets depend on the weight matrices. As $q$ varies, the shape and direction of the attractive set change and the optimal initial positions also move. Then the change in the shapes converges for sufficiently small $q$ in Figs. 3(c), 4(c) and 5(c), which concern more about control effort. In Figs. 3(a), 4(a), and 5(a), the shape of the contour is circle when the weight on state is relatively large. This implies that the optimal trajectory tends to take a shortest path to the origin for high energy trajectory. Actually, the distance to the origin is the shortest on the $x$-axis on the periodic solution. On the other hand, in Fig. 3(c), the attractive set for the case with $q=-3$ is ellipse which takes the optimal initial position 


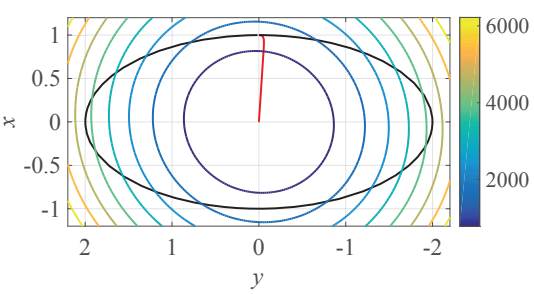

(a) $q=3$.

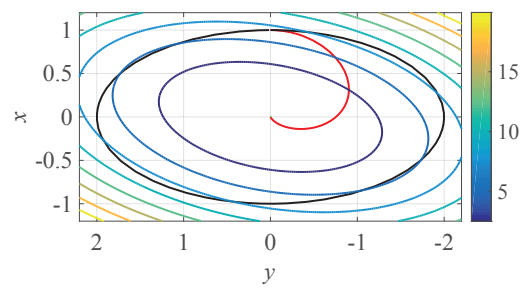

(b) $q=0$.

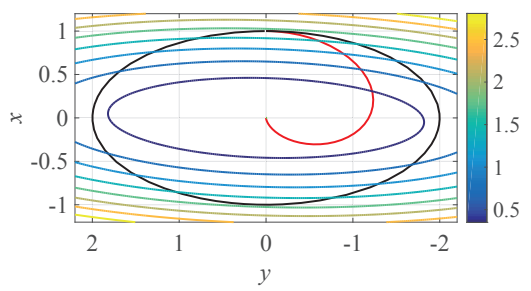

(c) $q=-3$.

Fig. 3. The trajectory departing from $(x, y)=(1,0): t_{f}=3$.

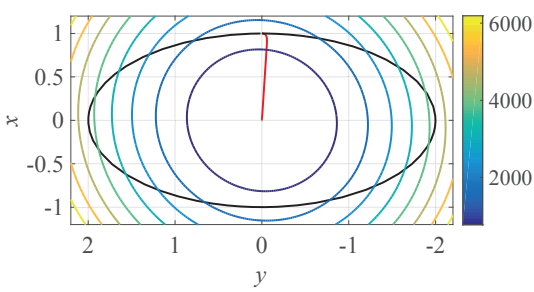

(a) $q=3$.

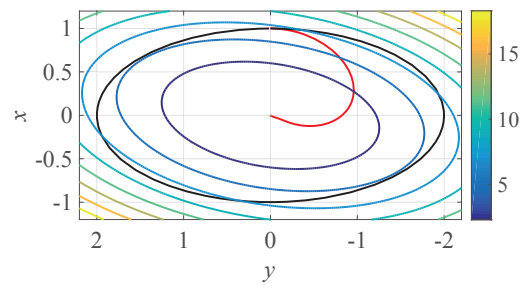

(b) $q=0$.

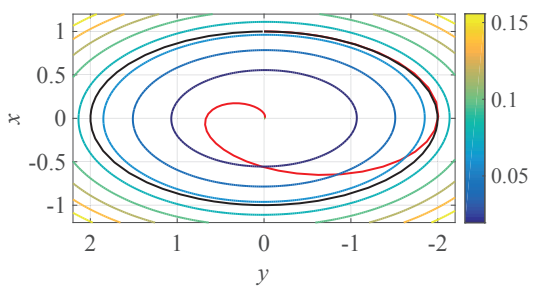

(c) $q=-3$.

Fig. 4. The trajectory departing from $(x, y)=(1,0): t_{f}=9$.

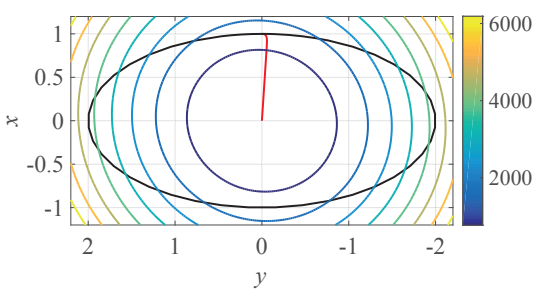

(a) $q=3$.

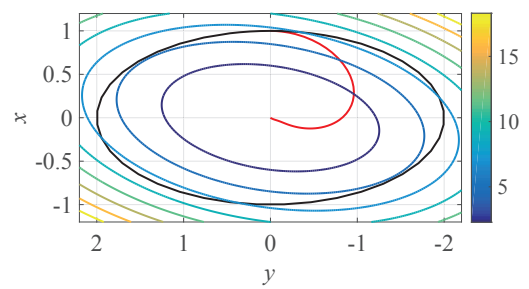

(b) $q=0$.

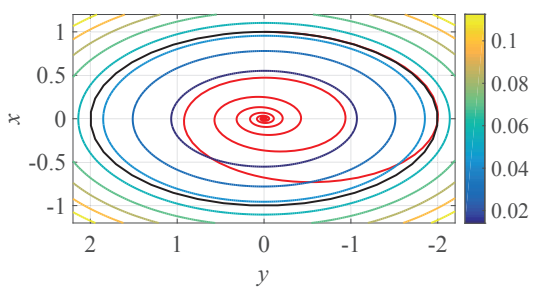

(c) $q=-3$.

Fig. 5. The trajectory departing from $(x, y)=(1,0): t_{f}=\infty$.

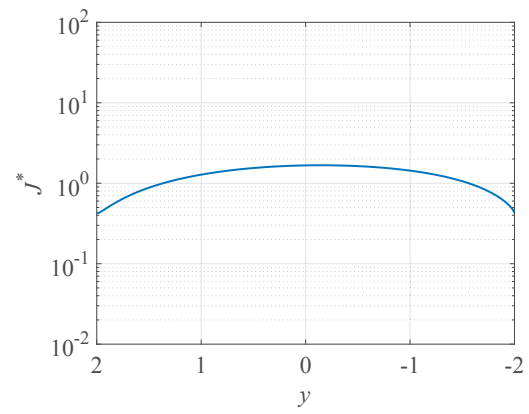

(a) $t_{f}=3$.

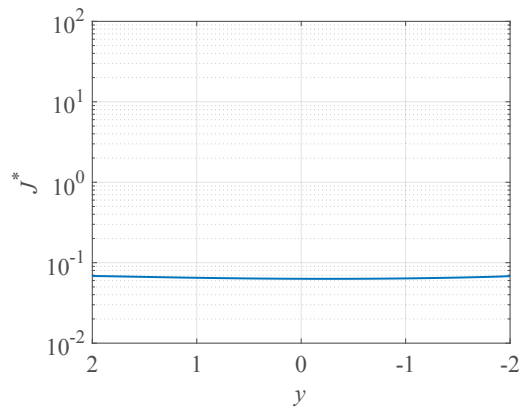

(b) $t_{f}=9$.

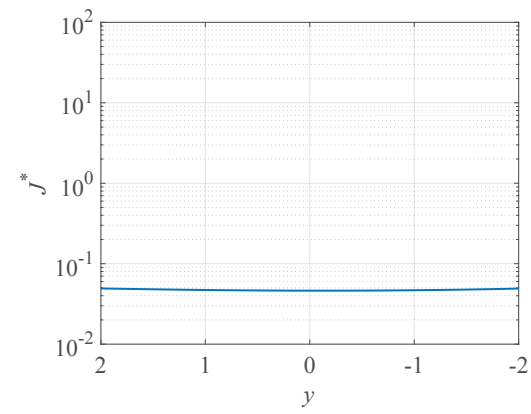

(c) $t_{f}=\infty$.

Fig. 6. Values of $J^{*}$ along initial orbit when $q=-3$.

on the $y$-axis. In this case, the time constraint is strong and the drift motion along $y$-axis might contribute to the optimal trajectory in this solution.

From Figs. 3(c), 4(c) and 5(c), it can be observed that the attractive sets converges to different shape for each $t_{f}$ even for the same $Q$ and $R$. Therefore, the final-time $t_{f}$ is also an important factor to determine the optimal trajectory for the finite-time problem. Moreover it can be said that the optimal solution is highly sensitive to the initial position for the short final-time problem from Figs. 3 - 5. In other words, the coast arc solution is obtained for fixed final-time problem to reduce the control effort while coasting arc is not important for infinite-time problem.

\subsection{Example of optimal trajectory design}

As an example, the optimal initial state is obtained analytically when $t_{f}=3$ and $q=-3$ in Fig. 6(a). The coefficient matrix of the attractive set obtained by Eq. (19) - (22) under 


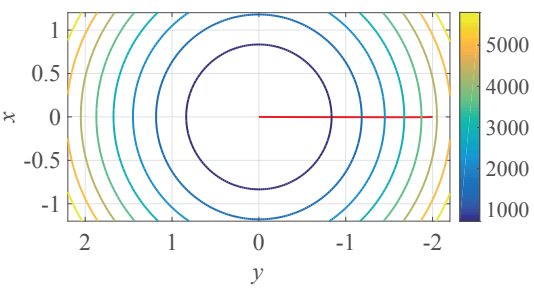

(a) $q=3$.

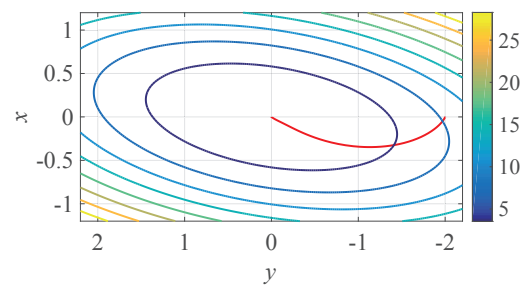

(b) $q=0$.

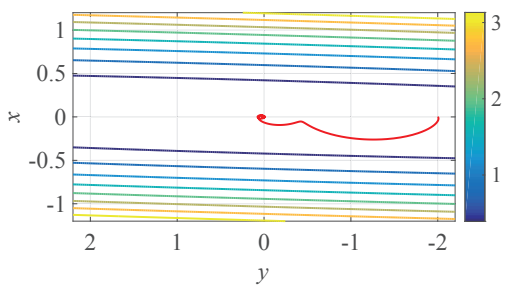

(c) $q=-3$.

Fig. 7. The contour of the attractive set for $x_{0}=[x, y, 0,0]$.

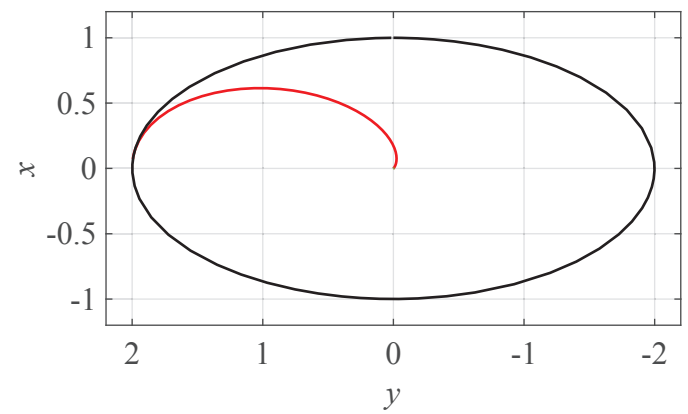

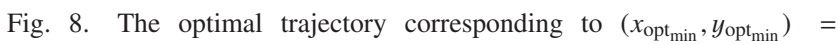
$(0.0723,1.9948)$.

periodic condition (B1) is given by

$$
X^{\prime}=\left[\begin{array}{cc}
1.6670 & -0.0451 \\
-0.0451 & 0.1071
\end{array}\right]
$$

From Eqs. (33) and (35), the optimal initial positions are given by

$$
\begin{aligned}
\left(x_{\mathrm{opt}_{\min }}, y_{\mathrm{opt}_{\min }}\right) & =( \pm 0.0723, \pm 1.9948) \\
\left(x_{\mathrm{opt}_{\max }}, y_{\mathrm{opt}_{\max }}\right) & =(\mp 0.9974, \pm 0.1446)
\end{aligned}
$$

Then, the minimum and maximum values of the performance index are given by Eq. (38) as

$$
C_{\text {min }}=0.4220, \quad C_{\max }=1.6735
$$

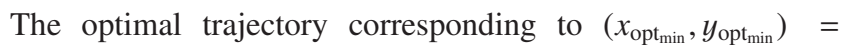
$(0.0723,1.9948)$ is shown in Fig. 8.

\subsection{Comparison of $L_{1}$-norm and rendezvous completion time}

We evaluate the trajectory using the $L_{1}$-norm, which is proportional to the fuel consumption. The $L_{1}$-norm is defined as follows:

$$
L_{1}=\int_{t_{0}}^{t_{f}}|\boldsymbol{u}(t)| d t
$$

and therefore the quadratic cost is not equivalent to the minimum fuel. However, it is known that the quadratic cost can be used to bound the $L_{1}$-norm. ${ }^{11)}$ We compare the optimal trajectories for finite-time, fixed final-state problem and infinite-time problem in terms of $L_{1}$-norm and flight time $t_{f}$.

The $L_{1}$-norm of the infinite-time problem approaches its infimum while flight time goes to infinity as $Q \rightarrow 0$. The infimum of the $L_{1}$-norm is approximately 0.62 as shown in Table 1 .

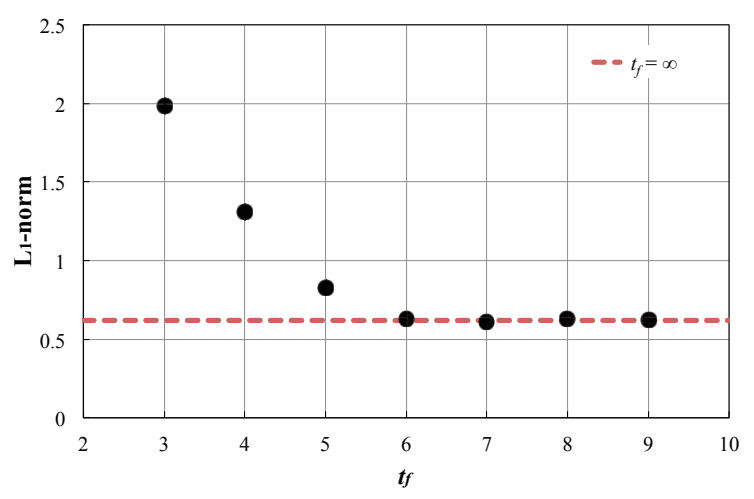

Fig. 9. $L_{1}$-norm and $t_{f}$ of the finite-time problem.

On the other hand, the $L_{1}$-norm of the finite-time problem approaches the infimum of infinite-time problem as flight time increases as shown in Fig. 9. However, the speed to approach infinite time problem is quite different for the $L_{1}$-norm and $t_{f}$ : the $L_{1}$-norm approaches that of the infinite-time problem around $t_{f}=9(\approx 1.4$ orbital period) from Fig. 9 and Table 1 . From this result, it can be said that the finite-time solution is more practical than infinite-time solution since $L_{1}$-norm is small with moderate flight time.

Table 1. Comparison of $L_{1}$-norm and $t_{f}$ between finite and infinite problems.

\begin{tabular}{rrr}
\hline & $t_{f}=9$ & $t_{f}=\infty$ \\
\hline$L_{1}$-norm & 0.63 & 0.62 \\
\hline
\end{tabular}

\section{Conclusion}

This paper presents the properties of an attractive set for optimal control for different boundary conditions using a linear quadratic regulator. A novel method for finding the optimal initial state based on the attractive set is proposed. One of the interesting property of the attractive set for the infinite-time problem is that it reflect the dynamical structure of the Hill's equations. The comparison of infinite-time problem and finite-time problem reveals that the finite-time solution is more practical than infinite-time solution since $L_{1}$-norm is small with moderate flight time.

\section{Appendix A: Angle of ellipse}

The following equation is assumed as an equation of an ellipse whose center is the origin at which the axis is rotating in 


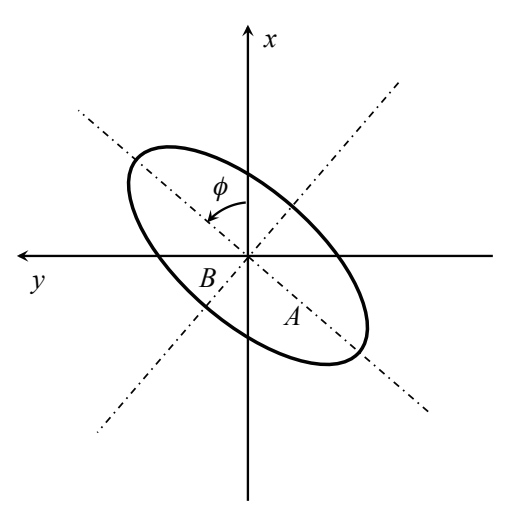

Fig. 10. Tilted ellipse at an angle $\phi$.

Fig. 10.

$$
a x^{2}+2 b x y+c y^{2}=1 \quad\left(b^{2}-a c<0\right)
$$

The rotation angle from the $x$-axis $\phi$ is given by

$$
\phi=\frac{1}{2} \arctan \frac{2 b}{c-a}
$$

where $\phi \in\left[-\frac{\pi}{4}, \frac{\pi}{4}\right]$.

In particular, the rotation angle of the major axis corresponds to Table 2 depending on the coefficients of the equation.

\begin{tabular}{l} 
Table 2. Angle of a major axis of an ellipse $\phi \in\left[-\frac{\pi}{2}, \frac{\pi}{2}\right]$. \\
\cline { 2 - 3 } \\
\begin{tabular}{cccc} 
& \multicolumn{3}{l}{ A } \\
& & $a \leq c$ & $\phi-\frac{\pi}{2}$ \\
& $a \leq 0$ & $a \geq c$ & $\phi+\frac{\pi}{2}$ \\
& $a \leq c$ & $\phi$ \\
\hline$b=0$ & $a>c$ & $\pm \frac{\pi}{2}$ \\
& $a<c$ & 0 \\
& $a=c$ & undefined. \\
\hline
\end{tabular}
\end{tabular}

\section{Proof.}

Assuming that the point moves to the coordinates $\left(x^{\prime}, y^{\prime}\right)$ when the point $(x, y)$ on the ellipse rotates by $\phi \in\left[-\frac{\pi}{2}, \frac{\pi}{2}\right]$ around the origin, the linear transformation is

$$
\begin{aligned}
& x=x^{\prime} \cos \phi+y^{\prime} \sin \phi \\
& y=-x^{\prime} \sin \phi+y^{\prime} \cos \phi
\end{aligned}
$$

Substitute this into Eq. (A1) of the ellipse to find $\phi$ which makes the coefficient of the term of $x^{\prime} y^{\prime}=0$.

$$
\begin{aligned}
& a\left(x^{\prime} \cos \phi+y^{\prime} \sin \phi\right)^{2} \\
& +2 b\left(x^{\prime} \cos \phi+y^{\prime} \sin \phi\right)\left(-x^{\prime} \sin \phi+y^{\prime} \cos \phi\right) \\
& +c\left(-x^{\prime} \sin \phi+y^{\prime} \cos \phi\right)^{2}=1 \\
\Leftrightarrow & \frac{1}{2}[(a-c) \cos (2 \phi)-2 b \sin (2 \phi)+a+c] x^{\prime 2} \\
& +\frac{1}{2}[-(a-c) \cos (2 \phi)+2 b \sin (2 \phi)+a+c] y^{\prime 2} \\
& +[(a-c) \sin (2 \phi)+2 b \cos (2 \phi)] x^{\prime} y^{\prime}=1
\end{aligned}
$$

Therefore, since the coefficient of $x^{\prime} y^{\prime}$ is 0 ,

$$
(a-c) \sin (2 \phi)+2 b \cos (2 \phi)=0
$$

it leads to

$$
\begin{aligned}
\tan (2 \phi) & =\frac{2 b}{(c-a)} \\
\sin (2 \phi) & =\frac{-2 b}{\sqrt{(a-c)^{2}+4 b^{2}}} \\
\cos (2 \phi) & =\frac{(a-c)}{\sqrt{(a-c)^{2}+4 b^{2}}} \\
\Leftrightarrow \phi & =\frac{1}{2} \arctan \frac{2 b}{(c-a)}
\end{aligned}
$$

The coefficients of $x^{\prime 2}$ and $y^{\prime 2}$ in Eq. (A3) become $1 / B^{2}, 1 / A^{2}$, respectively. Hence, the size of the major radius $A$ is given by

$$
\begin{aligned}
A & =\sqrt{\frac{2}{-(a-c) \cos (2 \phi)+2 b \sin (2 \phi)+a+c}} \\
& =\sqrt{\frac{2}{a+c-\sqrt{(a-c)^{2}+4 b^{2}}}}
\end{aligned}
$$

\section{Appendix B: The solution of Riccati equation with the con- dition of the periodic solution}

From the condition of the periodic solution of the Hill's equation, the velocity components $\dot{x}, \dot{y}$ on the periodic orbits can be represented by positions $x, y$ as

$$
\begin{aligned}
& \dot{x}=\frac{n y}{2} \\
& \dot{y}=-2 n x
\end{aligned}
$$

Therefore the 4-dimensional ellipsoid is reduced to the 2dimensional ellipse. Let $X$ be the coefficient matrix of an attractive set, then

$$
\begin{aligned}
J^{*} & =\boldsymbol{x}^{\mathrm{T}} X \boldsymbol{x} \\
& =\left[\begin{array}{ll}
x & y
\end{array}\right] X^{\prime}\left[\begin{array}{l}
x \\
y
\end{array}\right]
\end{aligned}
$$

where the new coefficient matrix is given by

$$
X^{\prime}=\left[\begin{array}{cc}
X_{11}-2 n X_{14}-2 n X_{41}+4 n^{2} X_{44} & X_{12}+\frac{n}{2} X_{13}-2 n X_{42}-n^{2} X_{43} \\
X_{21}+\frac{n}{2} X_{31}-2 n X_{24}-n^{2} X_{34} & X_{22}+\frac{n}{2} X_{23}+\frac{n}{2} X_{32}+\frac{n^{2}}{4} X_{33}
\end{array}\right]
$$

\section{References}

1) Kinoshita, H.: Celestial Mechanisms and Orbital Dynamics, Univ. of Tokyo Press, Tokyo, 1998, pp.22-31 (in Japanese).

2) Kyle, A., Srinivas, R. V., Pini, G., Jonathan, H., and Louis, B.: Spacecraft Formation Flying: Dynamics, control and Navigation, Butterworth-Heinemann, Oxford, 2009, pp. 83-90.

3) Kristiansen, R. and Nicklasson, J. P.: Spacecraft formation flying: a review and new results on state feedback control, Acta Astronautica, 65 (2009), pp. 1537-1552.

4) Shibata, M. and Ichikawa, A.: Orbital Rendezvous and Flyaround Based on Null Controllability with Vanishing Energy, J. Guidance, Control, and Dynamics, 30 (2007), pp. 934-945.

5) Guibout, V. M. and Scheeres, D. J.: Solving Relative Two-Point Boundary Value Problems: Spacecraft Formulation Flight Transfers Application, J. Guidance, Control, and Dynamics, 27 (2004), pp. 693 704. 
Trans. JSASS Aerospace Tech. Japan Vol. 17, No. 1 (2019)

6) Park, C. and Guibout, V. M. and Scheeres, D. J.: Solving Optimal Continuous Thrust Rendezvous Problems with Generating Functions, J. Guidance, Control, and Dynamics, 29 (2006), pp. 321 - 331

7) Ohtsuka, T.: Introduction to Nonlinear Optimal Control, Corona Publishing Co., Ltd., Tokyo, 2011, pp. 102-119 (in Japanese).

8) Anderson, B. and Moore, J. B.: Optimal Control: Linear Quadratic Methods, Courier Corporation, North Chelmsford, 2007, pp. 7-55.

9) Tsuneo, Y. and Imura, J.: Modern Control Theory, Shokoudou Press,
Tokyo, 1994, pp. 132-164 (in Japanese).

10) Bando, M. and Scheeres, D. J.: Attractive Sets to Unstable Orbits Using Optimal Feedback Control, J. Guidance, Control, and Dynamics, 39 (2016), pp. 2725-2739.

11) Gustafson, E. D., and Scheeres, D. J.: Optimal Timing of ControlLaw Updates for Unstable Systems with Continuous Control, J. Guidance, Control, and Dynamics, 32 (2009), pp. 878-887. 